\title{
LA STATICA DELLE CATTEDRALI GOTICHE E LA STATICA DEL DUOMO DI MILANO
}

\author{
LEONE CORRADI DELL'ACQUA (*)
}

RiassunTo. - Si illustrano i problemi statici sollevati dalla costruzione del Duomo di Milano e le soluzioni adottate per risolverli, sottolineando le similarità e le differenze che sussistono rispetto alle grandi cattedrali gotiche d'oltralpe. Il Duomo di Milano è stato concepito come una cattedrale del nord Europa, ma la sua costruzione è stata affidata prevalentemente a maestranze italiane, che avevano una abbastanza limitata confidenza con gli aspetti tecnici di questi monumenti. Di conseguenza, il Duomo di Milano si è avvalso solo parzialmente delle soluzioni tipiche delle cattedrali gotiche d'oltralpe e in molti casi sono state concepite soluzioni, il che fa del Duomo un monumento sotto molti aspetti unico anche dal punto di vista stutturale.

$* * *$

ABSTRACT. - The static problems raised by the construction of the cathedral of Milan and the structural solutions envisaged to face them are discussed, pointing out similarities and differences with respect to the great gothic monuments of northern Europe. Milan's cathedral was conceived as a northern gothic church, but its construction was carried on by Italian masons, little acquainted with the technical aspects of such monuments. As a consequence, northern gothic solutions were only partially adopted and completely new ones were worked up, so that the cathedral developed into a monument under several respects unique also from the structural point of view.

\section{INTRODUZIONE}

Le strutture monumentali giunteci dall'antichità, segnatamente le grandi cattedrali gotiche d'oltralpe, suscitano in noi profonda ammirazione, che deriva non solo dalle loro qualità artistiche, ma anche dall'imponenza della costruzione e dall'ardire dei costruttori. Tale ammi-

${ }^{(*)}$ Istituto Lombardo Accademia di Scienze e Lettere. Politecnico, Veneranda Fabbrica del Duomo-Milano. 
razione diviene ancora più grande se si considera quanto povero fosse il contesto di cultura tecnico-scientifica nel cui ambito queste opere sono state realizzate: non solo non esisteva una "teoria strutturale" adeguata, ma era difficile proporre una qualsiasi forma di "progettazione" dell'opera, che consentisse di prevederne il comportamento, valutarne l'adeguatezza e apportarvi le necessarie modifiche. Le tecniche di rappresentazione grafica erano infatti molto primitive, non esisteva un metodo unificato per la determinazione delle misure e l'uso dei numeri romani impediva l'esecuzione di calcoli anche semplici. In questa situazione, si potrebbe pensare che fosse diffuso il ricorso a modelli tridimensionali; questi sono stati a volte utilizzati nell'area italiana, anche se più per illustrare l'opera al committente che con fini progettuali, ma non risulta che all'epoca trovassero impiego al di là delle Alpi. ${ }^{1}$

I costruttori di queste cattedrali lavoravano a diretto contatto con le maestranze, interpretando contemporaneamente il ruolo di progettisti, di direttori di cantiere e spesso anche di scultori e di decoratori. La progettazione avveniva in maniera estemporanea, in genere evolveva in corso d'opera e si fondava su di un'esperienza acquisita con la pratica. Non esistevano testi o manuali capaci di trasmettere concetti, che configurassero l'esistenza di una vera "scienza del costruire": la cosa più simile a un "trattato" è un album di disegni di Villard de Honnecourt, architetto francese del XIII secolo, soprattutto inteso a fornire indicazioni per il taglio delle pietre in vista della realizzazione di particolari costruttivi di modesto impegno. ${ }^{2}$ Ciò non significa che non esistesse una competenza, empirica ma non per questo meno significativa, che si era andata sviluppando nel tempo e veniva trasmessa da maestro ad allievo. Una competenza esclusiva, quasi esoterica, condivisa da un certo numero di "maestri" che se ne sentivano i soli depositari.

Questa situazione contribuisce a spiegare il dibattito, sempre vivace e spesso molto acceso, che ha caratterizzato i primi anni della costruzione del Duomo di Milano. La realizzazione di questo monumento, dichiaratamente ispirato a forme gotiche transalpine, venne affidata a costruttori italiani, maestri Campionesi e Comacini, certamente esperti e collaudati artefici di grandi opere, cui tuttavia i coRoma, 1996.

1 C. Piga, Storia dei modelli: dal tempio di Salomone alla realtà virtuale, ENEL,

2 R. Bechmann, Villard de Honnecourt, architetto e ingegnere medioevale, ne: Il Medioevo, Le Scienze, quaderno n.36, pp. 44-51, Milano, 1987. 
struttori tedeschi e francesi non riconoscevano le competenze richieste da questa specifica architettura. A più riprese, a volte spontaneamente, più spesso sollecitati da autorevoli ambienti milanesi, questi maestri d'oltralpe si sono sentiti autorizzati a formulare critiche feroci nei confronti dell'operato dei costruttori italiani e previsioni catastrofiche sul destino della costruzione. Le risposte, sempre immediate e altrettanto decise, hanno provocato un contenzioso che si è sviluppato attraverso pubblici dibattiti, arena di confronto tra gli opposti schieramenti. I primi quindici anni della storia del Duomo sono punteggiati da questi eventi, che peraltro non hanno impedito alla costruzione di progredire con velocità ragguardevole. I maestri stranieri, benché godessero in genere dell'autorevole appoggio di Gian Galeazzo Visconti, prima signore e poi duca di Milano, riuscirono solo in poche occasioni e per brevi periodi a subentrare ai costruttori italiani. All'inizio del '400, con la morte di Gian Galeazzo, il contenzioso si spense, lasciando che la costruzione progredisse sotto la guida di maestri italiani. ${ }^{3}$ Questa matrice "italiana" ha un evidente riflesso nelle caratteristiche costruttive del Duomo di Milano, che si avvale solo parzialmente delle soluzioni statiche proprie delle grandi cattedrali d'oltralpe, cui peraltro il Duomo si richiama stilisticamente. D'altra parte, proprio questo riferimento a un'architettura sostanzialmente estranea alla tradizione italiana ha impedito di sfruttare le soluzioni concepite per le costruzioni romaniche e gotiche sorte in gran numero nella penisola. Il Duomo di Milano ha dovuto quindi elaborare soluzioni proprie, in alcuni casi brillanti, che ne fanno un monumento sotto molti versi unico anche da un punto di vista strutturale.

\section{LA STATICA DELLE GRANDI CATTEDRALI GOTICHE}

Si descrivono brevemente gli schemi statici su cui si reggono le grandi cattedrali gotiche d'oltralpe, schemi cui i costruttori si sono inevitabilmente riferiti. La loro comprensione richiede unicamente considerazioni di equilibrio, che, anche se non ancora compiutamente formalizzate all'epoca (la corretta legge per la composizione di più forze sarà stabilita solo alcuni secoli dopo), sono abbastanza semplici da far ritenere che i costruttori medioevali le possedessero quantomeno a livello intuitivo.

3 C. Ferrari da Passano, Il Duomo di Milano - Storia della Veneranda Fabbrica, NED, Milano, 1998. 
L'architettura gotica rappresenta il punto d'arrivo di un processo di alleggerimento della costruzione che si è gradualmente sviluppato lungo l'arco del Medio Evo. Le sue cattedrali sono caratterizzate da pilastri che, se confrontati con gli analoghi di epoche precedenti, appaiono molto snelli. Su di essi insistono sistemi di archi diversamente orientati, che sorreggono le volte: queste sono solo dei tamponamenti tra un arco e l'altro e non svolgono funzione portante (Fig. 1). Il problema fondamentale dell'architettura gotica è quindi quello di assicurare l'equilibrio di archi montati su pilastri.

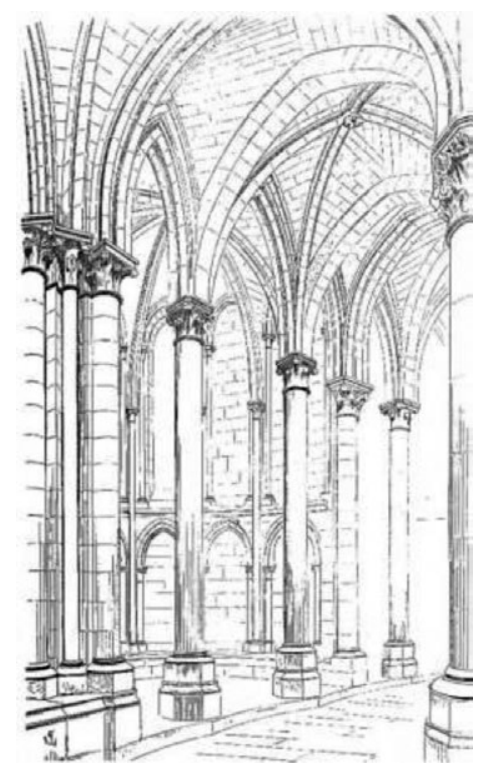

Fig. 1. Interno di cattedrale gotica. ${ }^{4}$

L'arco è stato usato fin dall'antichità per superare luci anche notevoli sollecitando il materiale prevalentemente a compressione, la condizione più favorevole. Per poter assolvere il suo compito, l'arco deve ricevere dai supporti, oltre che le forze verticali necessarie ad equilibrare il suo peso e i carichi sovrastanti, anche due forze orizzontali uguali e contrarie (in grigio in Fig. 2b); per il principio di azione e reazione, queste forze, cambiate

4 E. Viollet le Duc, Dictionnaire Raisonné de l'Architecture Française du XI au XVI Siècle, Parigi, 1854-1868. 
di segno, agiscono sul supporto, fornendo una spinta che tende ad allontanare tra di loro i punti di sostegno dell'arco. Se i supporti sono costituiti da pilastri non adeguatamente concepiti, la "spinta" può causarne il rovesciamento, con le conseguenze illustrate in Fig. $2 c$.

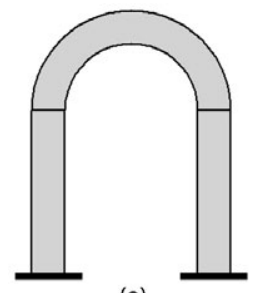

(a)

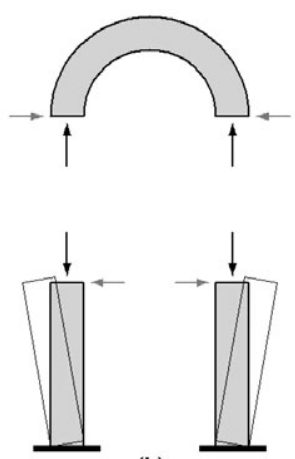

(b)

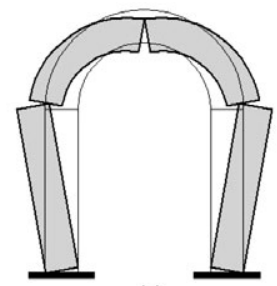

(c)

Fig. 2. Possibile effetto della spinta dell'arco sui pilastri che lo sostengono.

Diversi accorgimenti sono stati usati dai costruttori d'oltralpe per ridurre la spinta o contrastarne i suoi effetti. Il primo, talmente caratteristico dell'architettura gotica da passare quasi inosservato, si basa sulla constatazione che, a parità di altre condizioni, la spinta è tanto più elevata quanto più l'arco è ribassato. L'arco gotico, a sesto acuto, è "alto" $\mathrm{e}$, in quanto tale, esercita una spinta ridotta rispetto a quella che verrebbe esercitata da analoghi archi a tutto sesto.

La spinta permane tuttavia significativa. Essa è costituita dalla componente orizzontale della forza inclinata che l'arco scarica sul pilastro e, per assicurare l'equilibrio di quest'ultimo, questa forza deve essere "verticalizzata", in modo che la sua retta d'azione risulti sempre contenuta nella sezione del pilastro, in particolare nella sezione di base, la più critica. A questo fine si può fare parziale affidamento sul peso proprio del pilastro stesso: il peso di ogni concio, combinandosi con la forza proveniente dall'alto, dà luogo a una risultante più verticale della forza in ingresso (Fig. 3a). Il processo è facilitato se la sezione del pilastro viene aumentata man mano che ci si avvicina alla base (Fig. 3b), espediente spesso usato laddove consentito dal progetto. Ma è soprattutto la presenza di un'ulteriore forza verticale, come quella fornita dal peso di "guglie", statue o pinnacoli sovrastanti il pilastro, che fornisce un contributo significativo (Fig. 3c). Un peso aggiuntivo aumenta il li- 
vello di sforzo alla base, ma questo si mantiene comunque entro valori ampiamente accettabili: la rottura di un blocco di pietra o di marmo per l'esaurimento delle sue risorse in termini di resistenza è un evento così improbabile da non meritare di essere considerato, mentre il ribaltamento del pilastro rappresenta un'insidia concreta.

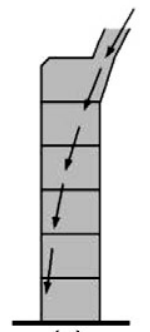

(a)

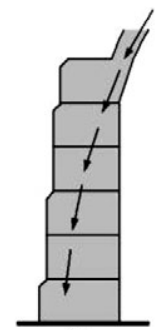

(b)

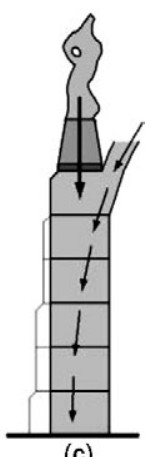

(c)

Fig. 3. Verticalizzazione della spinta per effetto del peso del pilastro e di un sovrastante carico aggiuntivo.

La soluzione di Fig. $3 b$ veniva di regola adottata per i pilastri posizionati in corrispondenza delle pareti laterali o dell'abside. I gradoni erano collocati all'esterno e fuoriuscivano dalla muratura, venendo a costituire i cosiddetti "contrafforti". Nel corso del tempo, la loro base si è notevolmente sviluppata in lunghezza, dando luogo a vere e proprie pareti che assolvevano il compito di scaricare a terra la spinta (Fig. 4). L'evoluzione del contrafforte ha prodotto quella che è forse la caratteristica più saliente delle cattedrali d'oltralpe, l'“arco rampante”. Ci si è infatti resi conto che un contrafforte molto sviluppato in lunghezza poteva essere alleggerito senza comprometterne il funzionamento. La parte della spinta che non poteva essere incanalata nel pilastro veniva convogliata in un arco inclinato, e trasferita più in basso, ad un altro pilastro, il contrafforte vero e proprio, eventualmente stabilizzato da una guglia (Fig. 5).

Unitamente ad archi a sesto acuto, molto rialzati, e a pilastri in pietra, pesanti anche se snelli, archi rampanti, contrafforti e guglie rappresentano gli ingredienti fondamentali delle cattedrali gotiche del nord Europa. Oltre ad assolvere importanti funzioni statiche, questi elementi caratterizzano in modo molto marcato l'aspetto esterno dell'opera, come illustrato dal celebre esempio di Fig. 6. 


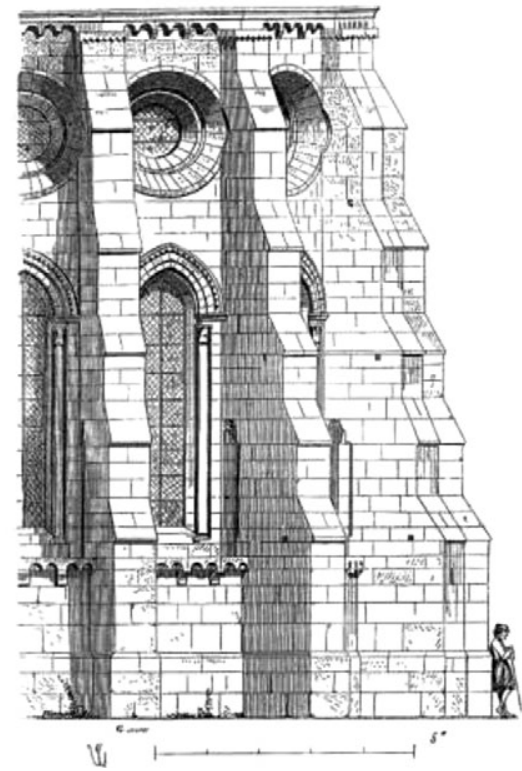

Fig. 4. Contrafforti. ${ }^{4}$

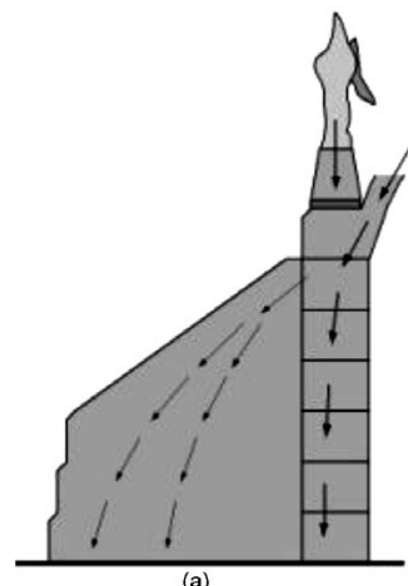

(a)

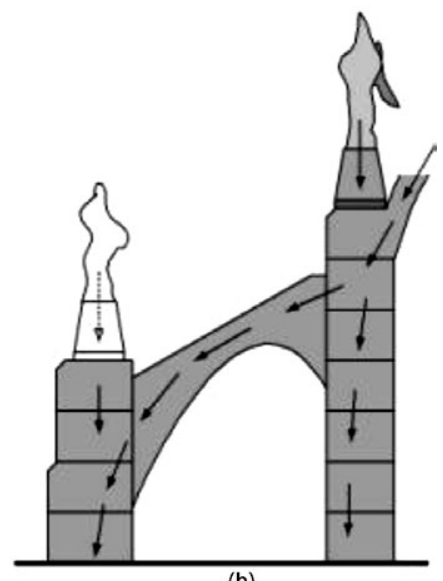

(b)

Fig. 5. Dal contrafforte all'arco rampante. 


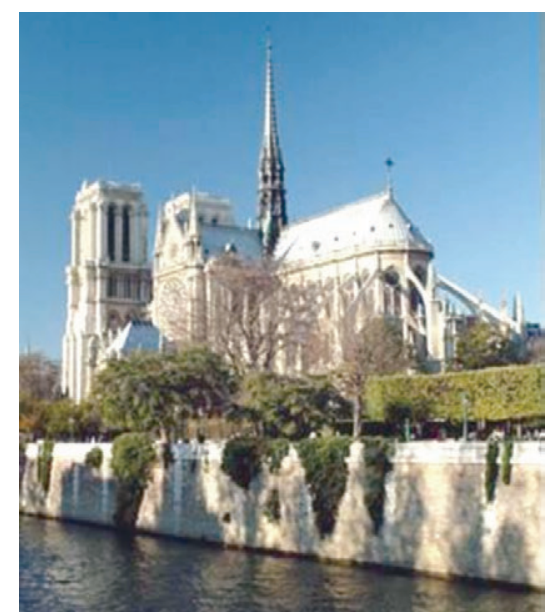

Fig. 6. Cattedrale di Notre Dame a Parigi bttp://en.wikipendia.org/wiki/Notre_Dame_de_Paris

\section{SULL'EFFETTIVA CULTURA STATICA DEI COSTRUTTORI}

E' quantomeno singolare che i concetti statici illustrati nel paragrafo precedente, benché relativamente semplici, non siano stati oggetto di trattazione sistematica. Non sembra troppo ardito pensare che se, per assurdo, queste cattedrali fossero state costruite dagli antichi greci qualcuno, per esempio un Archimede, non avrebbe mancato di produrre una trattazione del genere, ma di siffatte opere non si trova traccia in epoca medioevale. E' quindi spontaneo domandarsi se e quanto i costruttori fossero effettivamente consapevoli delle implicazioni statiche delle soluzioni da loro adottate.

A favore di una raggiunta e consapevole padronanza dei fondamentali concetti statici sembrano parlare le stesse cattedrali che oggi vediamo: costruzioni imponenti, che hanno sfidato i secoli e ancora trasmettono una sensazione di notevole solidità. Va però considerato che non tutte queste opere sono giunte fino a noi. Le cronache dell'epoca riportano notizie di crolli, anche clamorosi, come quello della cattedrale di Sens (1267), della guglia di Saint Bénigne a Digione (1272) e della volta della cattedrale di Bauvais (1284) in Francia e si ha notizia di analoghi incidenti verificatisi in Inghilterra e Germania. I crolli avvenivano per lo più in fase di costruzione, o comunque prima che questa fosse completata in tutti i suoi dettagli. Ciò sembrerebbe indicare che delle implicazioni statiche di alcuni elementi si avesse una consapevo- 
lezza solo parziale. Le guglie, ad esempio, venivano spesso posizionate per ultime, a volte anni dopo il completamento del corpo principale della costruzione, il che fa pensare che esse fossero ritenute soprattutto degli elementi decorativi e non si considerasse che in loro assenza la costruzione risultava più fragile.

Le cattedrali, molte, che hanno completato con successo la fase di costruzione sono giunte fino a noi. Ciò tuttavia non è necessariamente indice di una concezione strutturale particolarmente felice. $\mathrm{Si}$ tratta infatti di costruzioni molto "pesanti", che dedicano la quasi totalità del loro impegno a portare sé stesse. Sovraccarichi accidentali, quali quelli indotti dal vento o dalla neve, che possono rivelarsi critici in altre circostanze, hanno su di loro effetti marginali. Circa quarant'anni fa Heymann, in uno studio che interpretava il comportamento di questi monumenti alla luce del calcolo a rottura, ${ }^{5}$ dimostrava un teorema, per certi versi paradossale ma scientificamente corretto. Questo affermava che "se una cattedrale gotica resiste cinque minuti, resisterà cinquecento anni”, specificando peraltro che $\mathrm{i}$ cinquecento anni andavano intesi come un valore prudenziale, in quanto la teoria non prevedeva alcun limite superiore alla vita della struttura. In effetti, una volta superata la fase di costruzione e un transitorio iniziale (in verità, più di "cinque minuti") in cui l'assestamento del terreno può provocare sensibili cedimenti, le condizioni della cattedrale si mantengono sostanzialmente immutate. Il suo margine di sicurezza può anche essere molto ridotto ma, se non sopraggiungono fenomeni nuovi, non vi è motivo perché ad un certo punto si verifichi una crisi.

Il solo fatto che una cattedrale abbia evitato il collasso, quindi, non testimonia una particolare sapienza costruttiva. Del resto, esaminando i diversi monumenti, non è difficile riconoscere, accanto a realizzazioni staticamente coerenti, esempi in cui componenti potenzialmente in grado di assolvere compiti strutturali sono trattati alla stregua di elementi puramente decorativi. La Fig. $7 a$ illustra l'interno della cattedrale di Exeter, in Inghilterra: dai pilastri dipartono fasci di nervature, alcune delle quali formano archi di significato strutturale modesto, se non inutili. Nel chiostro della cattedrale di Gloucester

5 J. Heymann, The stone skeleton, International Journal of Solids and Structures, 2, pp.249-256, 1966. 
(Fig. 7b) non sopravvive neppure l'apparenza di una funzione strutturale delle nervature, ridotte a un puro ricamo superficiale. Ancora più stupefacente appare la volta in Fig. 8 dove le nervature, anziché dipartire da un pilastro e sorreggere la copertura, pendono da quest'ultima, con un effetto indubbiamente suggestivo, ma strutturalmente un poco inquietante. Queste realizzazioni venivano considerate una sorta di virtuosismo, suggerendo che i costruttori fossero in qualche misura consci di violare deliberatamente corretti concetti statici. Tuttavia, il solo fatto che esse fossero molto diffuse (gli esempi riportati si riferiscono all'Inghilterra, ma se ne trovano anche altrove) indica come l'aspetto statico non fosse considerato fondamentale.

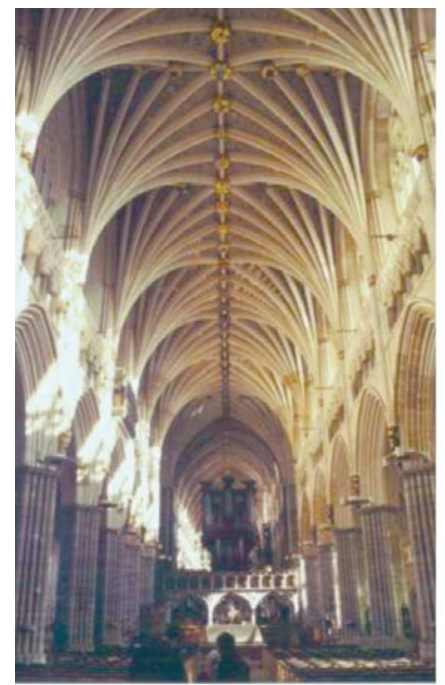

(a)

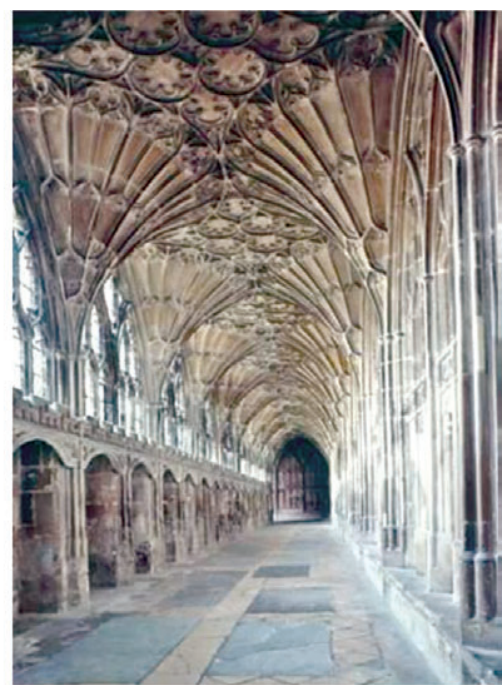

(b)

Fig. 7. (a) interno della cattedrale di Exeter (1328-1348), (b) chiostro della cattedrale di Gloucester (1381-1412).

(a) bttp://en.wikipendia.org/wiki/Image:ExeterCathedralNave.jpg (b) bttp://www.gloucestercathedral.org.uk

Il quesito riguardante l'effettiva consapevolezza delle implicazioni statiche dei diversi interventi rimane quindi aperto. I metodi di analisi strutturale oggi disponibili, se applicati alle costruzioni esistenti, permetterebbero di dare una risposta precisa. In effetti, le trattazioni di cui si è lamentata la mancanza in epoca medioevale, sono state prodotte nel XVIII secolo, ad opera di ingegneri francesi e ita- 
liani (si veda, a questo proposito, il trattato di Benvenuto, ${ }^{6}$ capitolo 9). Questi studi non avevano come oggetto le cattedrali gotiche, ma piuttosto le costruzioni dell'epoca, peraltro ancora realizzate in pietra e costituite fondamentalmente da archi e pilastri (oltre che da cupole). Essi si basavano comunque su concetti puramente statici, analoghi a quelli introdotti a livello intuitivo nel paragrafo precedente, ed erano quindi potenzialmente applicabili anche all'architettura medioevale. In effetti, un esame delle costruzioni gotiche alla luce di questi risultati è stato compiuto, tra gli altri, da Eugéne Viollet le Duc nella seconda metà dell'Ottocento (si veda nota ${ }^{7}$ e, soprattutto ${ }^{4}$, alle voci Construction, Arc-boutant e Contre-fort). Un'interpretazione del comportamento delle cattedrali in termini di calcolo a rottura è stato compiuto più recentemente da Heymann nell'articolo già citato. ${ }^{5}$

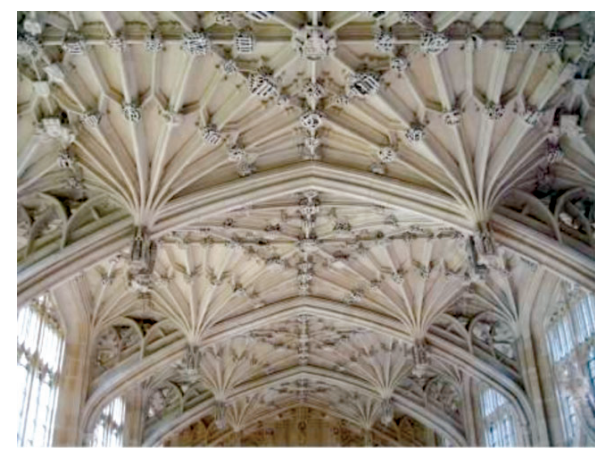

Fig. 8. Lierne vault, Divinity school, Oxford (1427-1483, arch. W. Orchard). bttp://www.flickr.com/photos/inel/458786687/

Questi studi sono di grande interesse perché, basandosi su considerazioni in larga misura accessibili ai costruttori medioevali, permettono una ricostruzione della loro concezione costruttiva. Peraltro, essi rivestono carattere molto generale e non risulta che siano stati utilizzati per esaminare il comportamento di specifiche costruzioni esistenti. Operazioni di questo genere sono state però effettuate assimilando le cattedrali a un solido elastico. Il procedimento suscita alcune perples-

6 E. Benvenuto, La Scienza delle Costruzioni e il suo Sviluppo Storico, Sansoni, Firenze, 1981.

7 E. Viollet le Duc, Entretiens sur l'architecture, Parigi, 1858-1872. 
sità, sia perché considerare come un solido "continuo" (in senso matematico) una costruzione in blocchi di pietra rappresenta un'approssimazione piuttosto ardita, sia perché la teoria dell'elasticità si basa in maniera essenziale su concetti legati alla deformabilità della struttura e del materiale, concetti che sono stati faticosamente sviluppati nel corso del settecento e appaiono del tutto estranei alla cultura medioevale.

Ciò non ha tuttavia impedito di ottenere risultati di interesse. Ad esempio, negli anni '70 Mark ha messo a confronto due celebri costruzioni francesi, le cattedrali di Chartres e di Bourges. ${ }^{8,9}$ La prima ha costituito un punto di riferimento per molte realizzazioni successive, che ne hanno ripreso le soluzioni statiche fondamentali, mentre la seconda rappresenta un caso praticamente isolato. Mark ha condotto sulle due costruzioni un'indagine sperimentale, valutando lo stato di sforzo locale con metodi di fotoelasticità. La conclusione, per certi versi sorprendente, è stata che il molto imitato schema di Chartres si rivela ben meno efficiente di quello della cattedrale di Bourges. Se ne dovrebbe concludere che i costruttori dell'epoca non fossero in grado di stabilire i meriti relativi delle due opere o, quantomeno, che non fossero interessati a farlo.

Quanto detto rende difficile attribuire ai costruttori del periodo gotico una profonda conoscenza dei concetti statici e una piena padronanza delle tecniche atte a metterli in pratica. E' peraltro evidente che, non potendo questi grandi monumenti essere sorti ed aver sfidato i secoli solo in virtù di circostanze quasi fortuite, chi li ha creati doveva possedere una solida competenza in materia, anche se in forma diversa da quella che con criteri moderni riteniamo corretta. Certamente non esisteva una teoria strutturale, intesa come insieme di concetti suscettibili di essere trasmessi ad altri. Non era neppure pensabile una progettazione sulla carta, che permettesse di prevedere il comportamento statico dell'opera, valutare comparativamente diverse soluzioni o apportarvi miglioramenti, e che potesse essere affidata alle maestranze per l'esecuzione. Il costruttore operava direttamente in cantiere e la progettazione procedeva di pari passo con la realizzazione. Le soluzioni adottate per risolvere i formidabili problemi statici che questi monumenti pone-

8 R. Mark, L'analisi strutturale delle cattedrali gotiche, Le Scienze, n. 54, febbraio 1973.

9 R. Mark, A. Borg, Chartres cathedral: a reinterpretation of its structure, The Art Bulletin, 55, pp.367-372, 1973. 
vano scaturivano da una combinazione di geniali intuizioni e di una cultura tecnica acquisita con l'esperienza. Ogni cattedrale felicemente portata a termine insegnava qualcosa, contribuendo a formare un bagaglio di conoscenza, certamente di natura empirica, ma non per questo di scarso significato.

Il desiderio di realizzare cattedrali sempre più imponenti ha portato ad applicare queste nozioni al di fuori degli ambiti collaudati, spesso con successo, a volte con inconvenienti anche seri. La crisi della volta della cattedrale di Bauvais ha di fatto posto fine alla "corsa verso l'alto" intrapresa dai costruttori. A questo punto, ogni nuova costruzione disponeva di modelli di riferimento. La vena creativa si è forse affievolita, rivolgendosi più agli aspetti decorativi che non a quelli strutturali, ma il bagaglio di conoscenze tecniche e costruttive accumulatosi con l'esperienza si rivelava del tutto adeguato, al punto da scoraggiare la ricerca di soluzioni migliori, di una cattedrale "strutturalmente ottimale". Per la loro stessa natura empirica e non formalizzata, queste conoscenze non potevano divenire patrimonio comune. Esse venivano acquisite operando in cantiere e nei cantieri venivano trasmesse da maestro ad allievo, restando confinate all'interno della cerchia dei costruttori. Questi hanno finito per formare gruppi elitari, che si sentivano depositari di una cultura costruttiva esclusiva, che altri non possedevano e a cui non avevano modo di accedere.

\section{LA STATICA DEL DuOmo Di Milano}

Questa situazione ha avuto riflessi importanti sul Duomo di Milano. La sua costruzione ha avuto inizio nel 1386, quando il gotico volgeva al tramonto nella penisola, e si richiamava volutamente alle forme transalpine, un'architettura comunque estranea alla tradizione italiana. Le ragioni di questa scelta sono da taluni attribuite alla volontà di Gian Galeazzo Visconti, da poco divenuto signore di Milano e di tutti i domini viscontei, un territorio molto vasto che una serie di fortunate campagne militari ha rapidamente ampliato fino a comprendere quasi tutta l'alta Italia e una buona parte dell'Italia centrale. Si pensa che Gian Galeazzo abbia vagheggiato l'idea di costituire uno stato nazionale, alla stregua di quelli che si andavano configurando al di là delle Alpi, con i Visconti come casa regnante e Milano come capitale, e questa capitale doveva essere dotata di una cattedrale che nelle dimensioni e nelle forme esteriori richiamasse le grandi cattedrali d'oltralpe. La realizzazione è stata affidata alla "Veneranda Fabbrica del Duomo", che è stata autorizzata a raccogliere contributi da parte dei cittadini. Questi sono 
stati inizialmente tanto cospicui che la fabbriceria ha rapidamente raggiunto una completa indipendenza economica. Ciò le ha conferito una notevole autonomia nei confronti dei poteri ecclesiastico e politico, incluso la stesso Gian Galeazzo.

Nonostante l'esplicita volontà di richiamarsi a modelli transalpini, la costruzione è stata affidata a maestranze italiane, maestri Comacini e Campionesi, di indubbia competenza costruttiva ma che in questa circostanza dovevano confrontarsi con problemi per essi non usuali. Nell'affrontarli, questi maestri hanno palesato non poche incertezze, tanto che la Veneranda Fabbrica ha dovuto provvedere a continui avvicendamenti. Ripensamenti e modifiche in corso d'opera erano all'ordine del giorno, cosa che tuttavia non ha impedito un progresso inizialmente molto rapido della costruzione.

La situazione non ha mancato di sollevare perplessità e autorevoli ambienti milanesi, primo tra tutti lo stesso Gian Galeazzo, hanno sollecitato il ricorso a maestri d'oltralpe. Uno di questi, Nicolas de Bonaventure, è stato chiamato già nel 1389 ad affiancare Simone d'Orsenigo. La sua permanenza, durata circa un anno, ha lasciato alcune tracce nella costruzione, segnatamente in alcuni particolari decorativi e, soprattutto, nei "finestroni" dell'abside, che denotano un'impostazione decisamente "francese" (Fig. 9). I rapporti con le maestranze italiane, tuttavia, non erano facili e le divergenze sono presto degenerate in aperti conflitti.

In effetti, i costruttori d'oltralpe non vedevano di buon occhio il fatto che la realizzazione di un'architettura dei cui segreti si sentivano depositari venisse affidata a maestranze italiane. Sfruttando gli incoraggiamenti che pervenivano dagli ambienti vicini a Gian Galeazzo, e a volte spontaneamente, sono spesso intervenuti, accusando apertamente i costruttori italiani di incompetenza e formulando previsioni funeste sulle sorti dell'edificio. Alcuni di questi maestri, come Ulrico di Fussingen, il principale artefice del Duomo di Ulm, erano personaggi di indubbia autorevolezza, il cui parere non poteva essere ignorato. I costruttori italiani, che in genere godevano dell'appoggio della Veneranda Fabbrica, rispondevano a queste accuse con immediatezza e precisione. Le questioni sfociavano in pubblici dibattiti, cui prendevano parte anche esperti esterni chiamati da entrambe le parti, e hanno avuto esito apparentemente alterno: Ulrico di Fussingen, ed Enrico da Gamodia prima di lui, hanno ottenuto l'incarico di architetti della Veneranda Fabbrica, ma sono stati rimossi dopo pochissimo tempo e la loro presenza non ha lasciato praticamente alcuna traccia nella costruzione. 


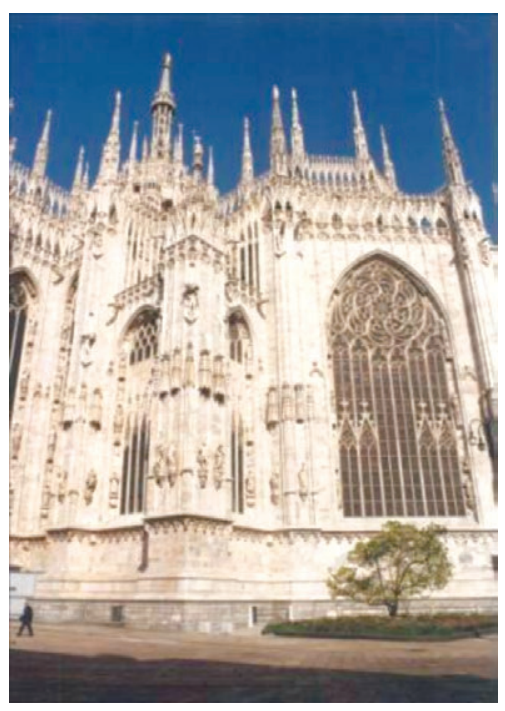

Figura 9, Un "finestrone" nell'abside del Duomo. (cortesia della Veneranda Fabbrica del Duomo).

L'ultimo atto di questa vicenda ha avuto inizio nel 1399, quando l'incarico di dirigere la costruzione è stato affidato a tre architetti francesi, tra cui il più autorevole era Jean Mignot, di Parigi. Questi ha immediatamente rivolto critiche estremamente severe a tutto l'operato precedente, sostenendo in particolare che il dimensionamento dei pilastri del tiburio era errato e le fondazioni insufficienti, e facendo intravedere la necessità di distruggere quanto fino ad allora realizzato per riprendere la costruzione dalle fondamenta. Al solito, la risposta da parte dei costruttori italiani non si è fatta attendere e la controversia è sfociata in un vero e proprio processo pubblico, con notai che verbalizzavano le argomentazioni presentate dalle due parti e dagli esperti da esse chiamati a supporto. Alla fine, malgrado le tesi francesi godessero dell'appoggio di Gian Galeazzo, la soluzione italiana è sopravissuta. Il Mignot non è stato immediatamente rimosso dall'incarico, ma la sua sorte era ormai segnata: poco dopo, prendendo a pretesto l'errata dimensione di un capitello (un errore tutto sommato veniale), è stato accusato di incompetenza e allontanato. In realtà, Jean Mignot incompetente non era e quando, negli anni '70 del secolo scorso, il Duomo ha evidenziato seri problemi statici, ci si è resi conto che le sue critiche, 
in particolare a riguardo del dimensionamento delle fondazioni, non erano affatto inconsistenti. ${ }^{10}$

Complice anche la morte di Gian Galeazzo, avvenuta poco dopo questi eventi, la costruzione è da allora proseguita sotto la guida di maestri italiani. Esperti d'oltralpe sono a volte intervenuti, ma adesso erano chiamati dalla Veneranda Fabbrica e operavano sotto il suo diretto controllo. Ne è risultata una cattedrale certamente gotica nel suo complesso, ma che si è avvalsa solo molto parzialmente delle soluzioni strutturali tipiche dei suoi modelli d'oltralpe. Sono ovviamente presenti archi ogivali e guglie, elementi che, pur non privi di funzioni statiche, sono indispensabili per conferire alla costruzione l'aspetto voluto. Il Duomo di Milano, tuttavia, non ha archi rampanti con funzioni strutturali, né contrafforti veri e propri, elementi completamente estranei alla cultura tecnica dei suoi costruttori. D'altro canto il Duomo è molto diverso dalle altre cattedrali italiane, il che ha impedito di sfruttare soluzioni collaudate in questo ambito. E' stato necessario elaborare soluzioni nuove, in alcuni casi molto felici e rimaste uniche, che fanno del Duomo un monumento di grande interesse anche dal punto di vista costruttivo.

Forse la più originale di queste soluzioni è rappresentata dal sistema detto delle "doppie volte" ${ }^{10}$ Come in tutte le costruzioni di questo tipo, le navate del Duomo di Milano corrono longitudinalmente e gli archi esercitano una spinta in direzione trasversale. A questa ossatura portante principale deve inevitabilmente essere sovrapposta una copertura, che nelle analoghe costruzioni transalpine, anche per far fronte alle notevoli precipitazioni nevose, è di regola costituita da un tetto a falde inclinate. Questo esercita una spinta aggiuntiva, modesta stante il non particolarmente elevato peso della copertura, che comunque si somma a quella fornita dagli archi portanti. Nel Duomo di Milano, la copertura è invece realizzata mediante volte a botte, molto ribassate, la cui sezione si vede in Fig. 10 sotto le falconature e le guglie in copertura. Queste volte corrono trasversalmente alle navate ed esercitano quindi la loro spinta in direzione longitudinale, senza aggiungersi alla spinta primaria. Inoltre (questo aspetto è meno evidente in figura) esse scaricano il loro peso direttamente sui pilastri che sostengono gli archi gotici, facendo sì che tutto il peso della copertura contribuisca alla verticalizzazione delle spinte, con un formidabile effetto stabilizzante.

10 C. Ferrari da Passano, Il Duomo Rinato, Veneranda Fabbrica del Duomo, 1988. 


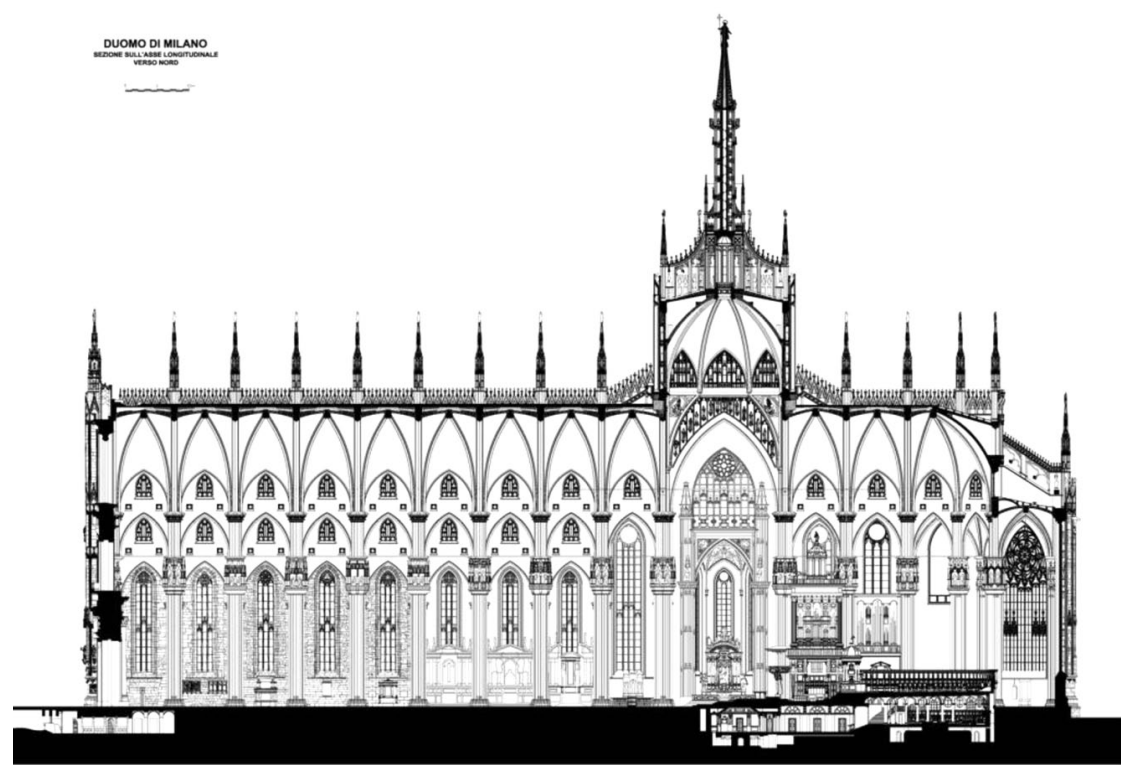

Fig. 10. Sezione longitudinale del Duomo di Milano. (cortesia della Veneranda Fabbrica del Duomo).

Si tratta di una soluzione che a mia conoscenza non ha precedenti, almeno in costruzioni di un certo impegno, e che neppure è stata ripresa in seguito, per cui deve essere considerata una caratteristica unica, esclusiva del Duomo di Milano. E' una soluzione per molti versi geniale e il fatto che essa sia stata concepita all'interno di un contesto relativamente povero di cultura tecnica (non vi è ragione di ritenere che i costruttori italiani fossero più smaliziati dei loro colleghi d'oltralpe) desta un indubbio stupore. Probabilmente, più che una consapevole scelta di natura statica essa è una conseguenza del desiderio di realizzare una copertura interamente pedonabile, che pure è una caratteristica unica del Duomo di Milano (Fig. 11). E' tuttavia innegabile che per ottenere questo risultato sia stata escogitata una soluzione di grande intelligenza statica, praticamente ottimale.

Si è detto in precedenza che il Duomo non presenta archi rampanti. L'immagine di Fig. 11 mostra alcuni elementi che si configurano come tali ma, considerando la loro collocazione, è facile constatare che essi non assorbono alcuna spinta significativa. Del resto il loro stesso aspetto, se confrontato con quello dei virili archi rampanti francesi, appare ben meno possente, quasi effeminato (Fig. 12). Questi archi indubbiamente contri- 
buiscono a conferire al Duomo l'aspetto di una cattedrale gotica, ma il loro compito essenziale sembra essere quello di sostenere le falconature.

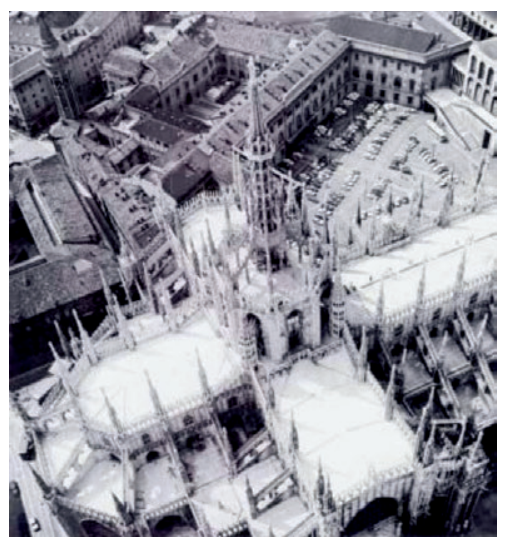

Fig. 11. Le terrazze del Duomo.

(cortesia della Veneranda Fabbrica del Duomo).

Se il sistema delle doppie volte rappresenta una soluzione strutturale particolarmente felice, altri interventi costruttivi sul Duomo di Milano non lo sono altrettanto. A volte, la ricerca di un compromesso tra la volontà di salvaguardare un aspetto esteriore gotico e la necessità di operare con tecniche familiari ai costruttori ha portato a vere e proprie forzature. Un esempio clamoroso è rappresentato dal tiburio, costruito nell'ultimo decennio del quattrocento, quando in Italia l'architettura del rinascimento si era ormai affermata. Un progetto, purtroppo perduto, del Filarete è stato scartato perché in contrasto con il carattere gotico del monumento, che la Veneranda Fabbrica riteneva prioritario salvaguardare. Il tiburio è stato realizzato su un progetto elaborato dall'Omodeo e dal Dolcebuono, che raggiungeva un buon compromesso tra le due esigenze. In particolare, dall'interno la cupola del tiburio sembra essere sostenuta da archi gotici (Fig. 13a), che però non hanno funzione portante: questa è svolta da quattro archi a tutto sesto, nascosti alla vista dal rivestimento gotico (Fig. 13b). Dal momento che gli archi gotici, preesistenti, erano centrati sui pilastri, gli archi portanti hanno dovuto essere posizionati "in falso", una soluzione non certo ineccepibile dal punto di vista strutturale. ${ }^{11}$

11 C. Ferrari da Passano, E. Brivio, Contributo allo studio del tiburio del Duomo di Milano, Arte Lombarda, $1^{\circ}$ semestre 1967. 


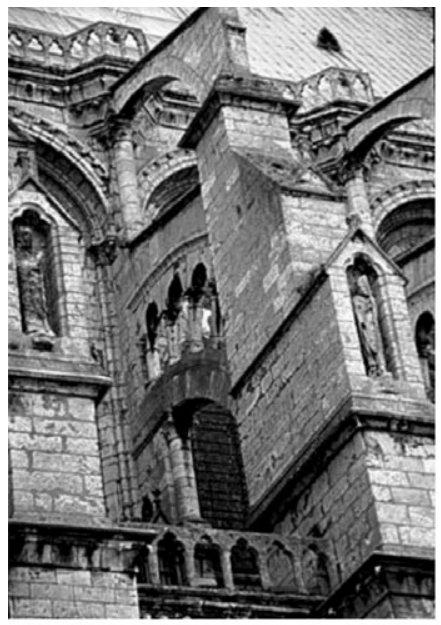

(a)

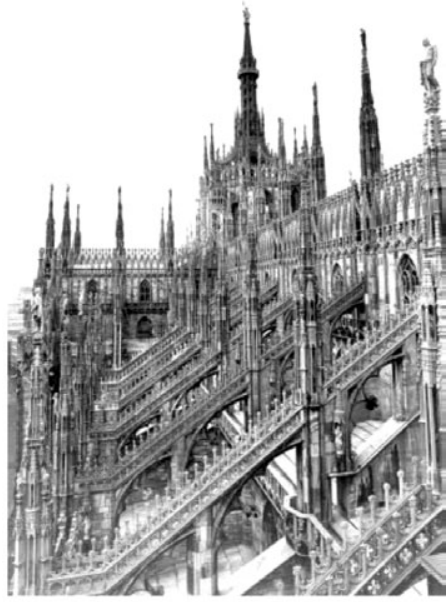

(b)

Fig. 12. Archi rampanti: (a) Notre Dame di Chartres, (b) Duomo di Milano. (a) bttp://docenti.lett.unisi.it; (b) cortesia della Veneranda Fabbrica del Duomo.

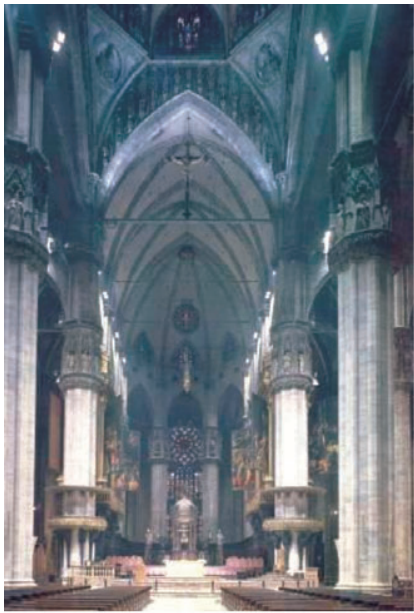

(a)

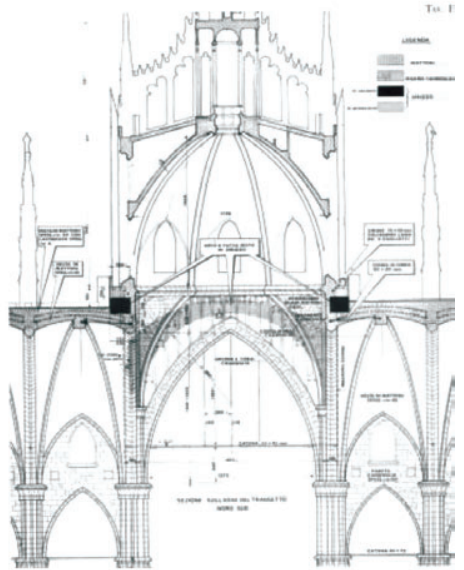

(b)

Fig. 13. Il tiburio del Duomo. (a) vista dall'interno; (b) particolare della struttura: uno degli archi a tutto sesto che sostengono il tiburio è evidenziato in grigio, la sezione dei due archi trasversali è in nero. (cortesia della Veneranda Fabbrica del Duomo). 


\section{CONCLUSIONE}

Il Duomo di Milano rappresenta un monumento sotto molti aspetti unico. La sua singolarità risiede principalmente nel fatto di essere un'architettura che si ispira a modelli estranei al contesto geografico-temporale cui appartiene, il che gli ha conferito il carattere composito che lo caratterizza e che non permette di ricondurlo all'interno di filoni codificati. E' difficile identificare dei suoi antenati tra le costruzioni che lo precedono, così come è difficile attribuire a costruzioni successive il diritto di essere definite sue discendenti. Questa sua natura è stata più volte sottolineata con riferimento agli aspetti architettonici e stilistici. In questa nota si è cercato di mostrare come essa sussista anche per gli aspetti strutturali e costruttivi.

Il Duomo di Milano può contare su molti entusiastici ammiratori, ma non mancano, e non sono pochi, i suoi detrattori. Questi giudicano la sua natura ibrida, piuttosto che composita, e lamentano una mancanza di purezza e coerenza stilistica. Questi giudizi critici possono essere estesi anche all'ambito strutturale: si è visto infatti come la necessità di confrontarsi con problemi insoliti abbia condotto i costruttori a elaborare soluzioni specifiche, a volte brillanti, ma a volte decisamente forzate, che un purista della statica si sente in dovere di criticare.

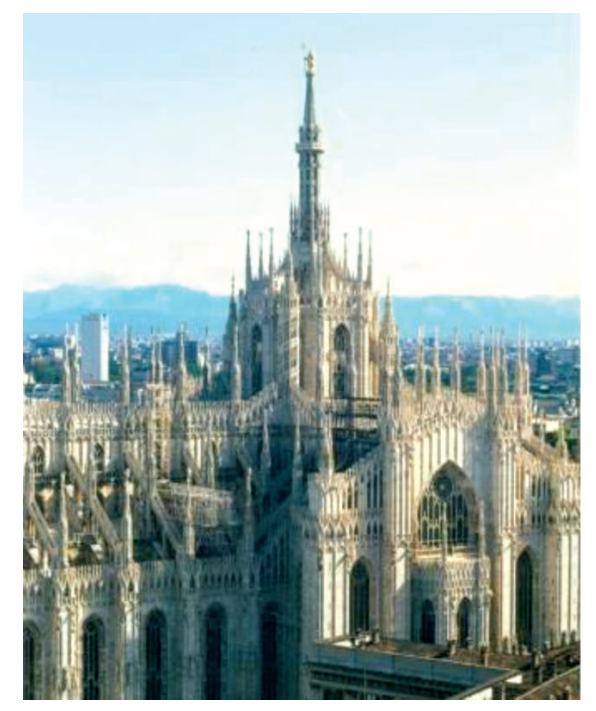

Figura 14. La guglia maggiore e l'esterno del tiburio. (cortesia della Veneranda Fabbrica del Duomo). 
Il tiburio rappresenta un esempio di questo tipo. Va peraltro considerato che l'aspetto strutturale non deve essere considerato (e certamente non era considerato all'epoca) come fine a sé stesso. Il tiburio del Duomo, sia visto dall'interno (Fig. 13a) che dall'esterno (Fig. 14) rappresenta un'opera di grande dignità architettonica e il fatto che questo involucro nasconda discutibili compromessi costruttivi non ne diminuisce certo il valore. A mio parere, il Duomo di Milano va giudicato nel suo complesso, senza soffermarsi troppo su dettagli che possono anche essere individualmente criticabili. Se visto in quest'ottica, esso appare un monumento ragguardevole, certamente degno della grande ammirazione che suscita.

\section{RINGRAZIAMENTI}

Per la stesura di questa nota mi sono avvalso della generosa collaborazione del compianto ingegnere Carlo Ferrari da Passano, per oltre quarant'anni Protoarchitetto della Veneranda Fabbrica e profondo conoscitore del monumento, che mi ha spiegato alcuni aspetti a me prima ignoti, in particolare il sistema delle doppie volte. A lui va la mia più sentita gratitudine. Sinceri ringraziamenti voglio anche esprimere a Roberto Fighetti, sempre disponibile ed efficace nell'aiutarmi a reperire il materiale iconografico relativo al Duomo. 
